\title{
Monocytes and neutrophils in the inflammatory cascade of systemic onset Juvenile Idiopathic Arthritis
}

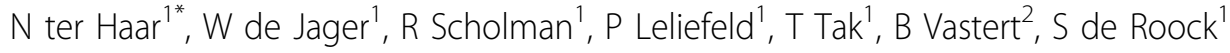 \\ From 8th International Congress of Familial Mediterranean Fever and Systemic Autoinflammatory Diseases \\ Dresden, Germany. 30 September - 3 October 2015
}

\section{Background}

Systemic onset Juvenile Idiopathic Artritis (sJIA), also known as Still's disease, is characterized by arthritis with symptoms of systemic inflammation such as spiking fever, rash and serositis. It is considered an autoinflammatory disease with a major role for the innate immune system, reflected by extremely high serum levels of S100 proteins and interleukin (IL)-18. How the number of monocytes and neutrophils relate to the increased levels of S100-proteins and IL-18 and to sJIA disease progression is still unknown.

\section{Objective}

To study the role of monocytes and neutrophils in the inflammatory cascade of sJIA.

\section{Methods}

We determined ex vivo cell frequencies and cell surface activation markers of sJIA patients at disease onset, in remission and healthy controls by flow cytometric analysis. For the in vitro assessment of neutrophils, we stimulated whole lysed blood or isolated neutrophils with S100-proteins, IL-18, platelet-activating factor (PAF) with or without Formyl-Methionyl-Leucyl-Phenylalanine (fMLP) or phorbol 12-myristate 13-acetate (PMA) and determined intracellular ROS production, degranulation and apoptosis. To investigate the role of monocytes, we stimulated peripheral blood mononuclear cells (PBMCs) from sJIA patients and healthy controls with S100-proteins (+/- ATP) or other TLR-ligands and determined the concentration of cytokines in the supernatant by multiplex immunoassays.

'University Medical Center Utrecht, Laboratory for Translational Immunology, Utrecht, Netherlands

Full list of author information is available at the end of the article

\section{Results}

Patients with new onset sJIA had significantly elevated neutrophil counts compared to healthy controls and sJIA patients with clinically inactive disease, while the amount of monocytes was not significantly different between the groups. Neutrophils from new onset sIIA patients showed an activated phenotype, reflected by higher ex vivo expression of Fc-gamma receptors (CD32 and CD64), markers of secretory vesicles (CD35) and specific granules (CD66b) compared to healthy controls. Neutrophils from new onset sJIA showed enhanced ROS production and degranulation and appeared to be more resistant to apoptosis. In contrast to the hyperactivated status of neutrophils in active sJIA,, PBMCs from these patients produced less IL-18 upon S100 stimulation compared to PBMCs from the same patient in remission or healthy controls. The same trend was observed when PBMCs from sJIA patients were stimulated with LPS, TLR2- or TLR7/8 ligands, suggesting cross-tolerance in these patient cells.

\section{Conclusions}

Although monocytes from sJIA patients with active disease are less responsive towards stimulation, neutrophil counts, ROS production and degranulation are clearly elevated. The exact role of each cell type and activity and their interaction in sJIA pathology is currently under investigation.

\footnotetext{
Authors' details

${ }^{1}$ University Medical Center Utrecht, Laboratory for Translational Immunology, Utrecht, Netherlands. ${ }^{2}$ University Medical Center Utrecht, Department of Pediatric Immunology, Utrecht, Netherlands.
}

Published: 28 September 2015 


\section{doi:10.1186/1546-0096-13-S1-054}

Cite this article as: ter Haar et al:: Monocytes and neutrophils in the inflammatory cascade of systemic onset Juvenile Idiopathic Arthritis.

Pediatric Rheumatology 2015 13(Suppl 1):054.

Submit your next manuscript to BioMed Central and take full advantage of:

- Convenient online submission

- Thorough peer review

- No space constraints or color figure charges

- Immediate publication on acceptance

- Inclusion in PubMed, CAS, Scopus and Google Scholar

- Research which is freely available for redistribution

Submit your manuscript at www.biomedcentral.com/submit 